\title{
The Effects of Priming Intermittent Theta Burst Stimulation on Movement-Related and Mirror Visual Feedback-Induced Sensorimotor Desynchronization
}

Jack Jiaqi ZHANG

Hong Kong Polytechnic University

Kenneth NK Fong ( $\nabla$ rsnkfong@polyu.edu.hk)

Hong Kong Polytechnic University Faculty of Health and Social Sciences https://orcid.org/0000-00015909-4847

Research article

Keywords: Theta burst stimulation, event-related desynchronization, metaplasticity, motor cortex, mirror visual feedback

Posted Date: August 24th, 2020

DOl: https://doi.org/10.21203/rs.3.rs-59384/v1

License: (c) (1) This work is licensed under a Creative Commons Attribution 4.0 International License. Read Full License 


\section{Abstract}

Background: The potential benefits of priming intermittent theta burst stimulation (iTBS) with continuous theta burst stimulation (cTBS) have not been examined in regard to sensorimotor oscillatory activities recorded in electroencephalography (EEG).

Objective: The objective of this study was to investigate the modulatory effect of priming iTBS (cTBS followed by iTBS) delivered to the motor cortex on movement-related and mirror visual feedback (MVF)induced sensorimotor event-related desynchronization (ERD), compared with iTBS alone, on healthy adults.

Methods: Twenty participants were randomly allocated into Group 1: priming iTBS - cTBS followed by iTBS, and Group 2: non-priming iTBS - sham cTBS followed by iTBS. The stimulation was delivered to the right motor cortex daily for 4 consecutive days. EEG was measured before and after 4 sessions of stimulation. Movement-related ERD was evaluated during left-index finger tapping and MVF-induced sensorimotor ERD was evaluated by comparing the difference between right-index finger tapping with and without MVF.

Results: After stimulation, both protocols increased movement-related ERD and MVF-induced sensorimotor ERD in high mu and low beta bands, indicated by significant time effects. A significant interaction effect favoring Group 1 in enhancing movement-related ERD was observed in the high mu band $\left(F_{(1,18)}=4.47, p=0.049\right)$, compared with Group 2 .

Conclusion: Our experiment suggests that priming iTBS with cTBS delivered to the motor cortex yields similar effects with iTBS alone on enhancing sensorimotor activation induced by movement and MVFbased observation on healthy adults. Further studies are warranted.

\section{Background}

Theta burst stimulation (TBS) is an accelerated form of repetitive transcranial magnetic stimulation (rTMS), which has been extensively employed in human studies after the first human experiment [1]. Using repetitive short bursts of high frequency stimulation (e.g., $50 \mathrm{~Hz}$ ), given five times per second, TBS is able to modulate corticomotor excitability, as measured by the amplitude of motor evoked potential (MEP) [1]. TBS given in an intermittent manner - intermittent theta burst stimulation (iTBS) - can lead to a facilitatory effect on the stimulated cortex, while TBS given in a continuous manner - continuous theta burst stimulation (CTBS) - does the opposite [2]. However, substantial response variability to TBS among humans has been noted in the previous literature [3]. Although TBS is an accelerated form of excitatory rTMS that may lead to superior clinical outcomes, a recent experiment showed that the response rate to iTBS or cTBS (i.e., the percentage of participants who presented increased or decreased MEP upon completion of the stimulation) is around $60 \%$ [4] and did not improve along with more delivered doses of the same stimulation, indicating that TBS has no effects on a substantial number of subjects. The inconsistency of the response to TBS may limit its utility in both research and clinical interventions [5]. 
Numerous biological factors that can influence the response to TBS have been reported; one of the adjustable factors is the history of neuronal activities [3]. Synaptic plasticity is regulated by previous neuronal activities via metaplasticity. Metaplasticity is a neuroprotective mechanism that modulates the threshold of synaptic plasticity to ensure that the neural system cannot be predominated by long-term potentiation (LTP) or long-term depression (LTD) [6]. Brain response to rTMS is likely to be influenced with the metaplastic mechanism. As an example, an excitatory rTMS protocol may fail to facilitate corticomotor excitability (i.e., LTP-like neuroplasticity) when the neuronal activities are already at a high level before the stimulation commences. Considering the mechanism of metaplasticity, several priming stimulation protocols, designed with a priming session followed by a stimulation session, have been investigated in healthy adults, with the aim to harness metaplastic mechanisms for potentiating the effects of rTMS [7]. Theoretically, an inhibitory priming session using cTBS is likely to make the brainstate more amenable to the facilitatory effects of iTBS, thereby delivering a stronger synergistic effect. In healthy individuals, this inhibitory priming session seems to amplify the facilitatory effect of iTBS, in contrast to iTBS alone, as reflected by the amplitude of MEP $[8,9]$. Utilizing the potential metaplasticity is likely to increase the effects of TBS, thus improving its clinical utility in populations with diseases [10].

The potentiating effect of priming iTBS has only been proven with lines of evidence of TMS-based metrics, such as MEP and short-interval intracortical inhibition (SICI) $[8,9,11]$. However, TMS-based metrics may not represent all cortical responses, reflecting exclusively those destined to the spinal cord [12]. And the magnitude of TMS-based metrics is also contaminated by the neuronal responses at subcortical and spinal levels, as well as the peripheral MEP [13], since a suprathreshold stimulation intensity is often used for the measurements. Electroencephalography (EEG) is a non-invasive measure of the electric activity of cortical neurons [14]. Sensorimotor event-related desynchronization (ERD) is thought to be a neurophysiological marker of sensorimotor activation, which could be induced through either movement execution and movement observation [15]. Movement-related sensorimotor ERD in both mu (i.e., central alpha, 8-12 Hz) and beta $(12-30 \mathrm{~Hz})$ rhythms was first reported by Pfurtscheller et al. [16]. During unilateral hand movement, movement-related sensorimotor ERD was found to be prominent in the hemisphere contralateral to the moving hand when preparing to move, and it expands bilaterally when executing the movement [15].

Sensorimotor ERD can also be viewed when observing the movement without overt movement execution, which is attributed to an assumed function of the human mirror neuron system (MNS) $[17,18]$. MNS is a class of the neuronal population that discharges during movement observation and execution. MNS was first found in the premotor and parietal areas of macaque monkeys [19] and numerous neuroimaging studies in humans have reported consistent neural activation over frontal-parietal areas in response to movement observation, indicating that there exists a homological neural system in humans (i.e., human MNS) [20]. There is functional connectivity between the MNS and primary sensorimotor cortex [21] and the downstream modulatory activity of the MNS on the primary sensorimotor cortex could be indexed by observation-induced sensorimotor ERD [18]. Mirror visual feedback (MVF) has been widely used in studies examining observation-induced sensorimotor ERD in healthy adults [22-24] and has also been utilized as a therapeutic form of intervention for the rehabilitation of upper limb motor functions after 
stroke (i.e., mirror therapy) [25]. In the MVF paradigm, mirror apparatus is placed at the midsagittal plane of the participant. Participants are instructed to perform unilateral hand movements while simultaneously viewing the MVF of their moving hand from the mirror. It has been reported that MVF could lead to a shift of sensorimotor ERD from the hemisphere ipsilateral to the moving hand [22-24]. Therefore, MVFinduced sensorimotor ERD is a useful index to study observation-induced sensorimotor activation and the involvement of MNS, which is potentially correlated with the capacity of motor learning from movement observation. This paradigm also allows us to study the activation of sensorimotor area when the corresponding upper extremity remains static, thus becoming potentially useful in studying the sensorimotor plasticity in patients with severe upper extremity disability such as stroke [26].

Sensorimotor ERD can be used to probe cortical oscillatory activities of large number of neurons in different rhythms, which would provide new insight on the sensorimotor plasticity induced by priming iTBS. A previous study comparing the effects of TBS on MEPs and movement-related rhythms showed that the modulatory effect of TBS was more reliable on movement-related ERD than that on MEPs [12]. To date, no study has explored the differential effects of priming iTBS (i.e., cTBS followed by iTBS) and iTBS on sensorimotor ERD. Hence, our study aims to investigate the neuromodulatory effect of priming iTBS on movement-related and MVF-induced sensorimotor ERD, compared with non-priming iTBS, on healthy adults. We hypothesized that both protocols could enhance the sensorimotor ERD induced by either movement or MVF-induced observation, and priming iTBS with cTBS could yield a stronger facilitatory effect, comparing with iTBS alone.

\section{Materials And Methods}

\section{Participants}

Potential participants were recruited from a local university. Twenty young healthy participants (Group 1: age $=27.40 \pm 2.07$, two women and eight men; Group 2: age $=27.10 \pm 2.08$, two women and eight men) were recruited. All of them were postgraduate and met all of the following criteria: 1) 18 to 30 years old; 2) right-handed, according to the Edinburgh handedness inventory [27]; and 3) normal or corrected-to-normal vision. Participants were excluded if they met any of the following criteria: 1) any contraindication to TBS, such as a history of seizures, metal implants, and pregnancy. All participants were screened by a standard safety checklist before enrollment [28]; 2) previous history of any neurological or psychiatric diseases; 3 ) presence of upper limb injuries in the past three months; and 4) presence of congenital deformities of the bilateral upper extremities. This study was approved by the Human Subjects Ethics Committee, The Hong Kong Polytechnic University (reference number: HSEARS20190326003). All participants voluntarily consented to participate in this study and their written informed consent was obtained before participation commenced.

\section{Experimental procedure}

Participants were randomly allocated to one of the following two groups by drawing lots: Group 1: cTBS followed by iTBS; and Group 2: sham cTBS followed by iTBS. All participants had to attend four 
consecutive TBS sessions and two EEG assessments before and immediately after 4 daily sessions of stimulation.

\section{Motor threshold assessment}

The stimulation site for iTBS was the right motor cortex. The optimal position was defined as the coil position eliciting the largest MEP, with the coil rotated $45^{\circ}$ from the sagittal plane. The stimulation position was maintained by a neuro-navigation system (Localite, Bonn, Germany). Resting motor threshold (RMT) is defined as the minimum intensity over the hot spot that elicits an MEP of no less than $50 \mu \mathrm{v}$ in three out of six trials over the contralateral first dorsal interosseous (FDI). MEPs were visualized and measured through the MEP monitor (MagVenture, Denmark), with an inter-pulse interval of at least five seconds.

\section{iTBS sessions}

Daily serial sessions of iTBS were delivered by MagPro X100 stimulator (MagVenture, Denmark) with a standard butterfly-shape coil (C-B60), over the right M1 for four consecutive days. Repeated applied stimulation sessions were used in order to obtain observable modulatory effects and to imitate the design usually applied in clinical intervention [29] .We followed previous studies, using four daily sessions of iTBS for healthy adults [30-32]. The standard 600-pulse TBS protocol was followed [2]. The stimulation intensity of iTBS was set at a safety limit of $70 \%$ of the individual's RMT [33]. We did not set the intensity based on the active motor threshold (AMT), because a previous study has shown that pre-stimulation muscle contraction during the measure of AMT could influence the after-effects of TBS [33]. Sham stimulation was delivered using the same coil that delivers only $20 \%$ of the individual RMT. All participants were told that TBS was a subthreshold stimulation that could not induce significant arm movements or somatosensory perception. The interval between priming and stimulation sessions was 10-minute. We choose the 10-minute interval based on a previous in vitro study about reversal of synaptic plasticity in response to TBS [34], and followed a previous human neurophysiological study about priming iTBS [8]. Participants were asked to complete a questionnaire regarding the side effects of iTBS they experienced upon the completion of each stimulation session.

\section{EEG acquisition}

EEG was captured with a 64-channel cap, using a Digital DC EEG Amplifier and Curry 7 (Compumedics Neuroscan, USA). Electrode impedance was kept below $10 \mathrm{kOhm}$ and the signal was sampled at $1000 \mathrm{~Hz}$. Participants were seated upright in an electromagnetic shielded room and required to minimize any body movements during the recording. Movement-related and MVF-induced sensorimotor ERD were evaluated. Left index finger tapping and incongruent (i.e., mirrored) visual observation of the right index finger tapping were used to elicit the ERD over bilateral sensorimotor areas, with a possibly right dominant lateralization [32,35]. For movement-related ERD, participants were instructed to tap on a computer keyboard three times with their left index finger, in response to 60 auditory cues (i.e., 300-ms beep sounds) delivered at random intervals (from seven seconds to 10 seconds) and to relax the finger after 
the completion of the movement. Participants were asked to focus on a centrally located fixation cross in a computer monitor placed in front of them.

For MVF-induced ERD, participants were asked to tap on a computer keyboard three times with their right index finger, in response to 60 auditory cues delivered at random intervals (from seven seconds to 10 seconds), and to relax the finger after completing the movement (Reference to finger tapping tasks). Movements were performed under two conditions. 1) A mirror view of the movement: Participants performed right-index tapping while simultaneously looking at the MVF of their moving finger. The MVF was created using a physical mirror $(406 \times 432 \mathrm{~mm})$ placed over their midsagittal plane, between both arms. A black curtain was used to block the view of their moving hand. 2) A direct view of the movement: Participants performed right-index tapping while looking at the direct visual feedback (DVF) of their moving finger. Their left hand was hidden by a non-transparent board (see Figure 1 for the EEG set-up). The order of conditions was randomized by drawing lots. A total of 60 movements were collected for each condition, with 180 movements in total.

\section{EEG time-frequency analysis}

Clean trials were analyzed in a time-frequency domain. The event-related spectral perturbation (ERSP) method was used to compute ERD power [36]. The power was baseline corrected. We selected a baseline period from $-1500 \mathrm{~ms}$ to $-1000 \mathrm{~ms}$ for correction, to avoid the contamination of neural oscillations caused by auditory cues delivered prior to the execution of movement. Subsequently, the power was averaged across all included trials and converted to log power (see the following formula).

$$
\operatorname{ERSP}(f, t)=\frac{1}{n} \sum_{k=1}^{n}\left(F_{k}(f, t)^{2}\right)
$$

where $\mathrm{n}$ is the number of trials, and $F_{k}(f, t)$ is the spectral estimation of the kth trial at frequency $f$ and time $t$. ERD was further computed using the following formula [37]:

$$
\text { ERD power }=\frac{1}{N} \sum_{f \in F} \sum_{t \in T}(E R S P(f, t))
$$

where $F$ represents the frequency band of interest. We defined four frequency bands of interest, including mu-1 (8-10 Hz), mu-2 (10-12 Hz), beta-1 $(12-16 \mathrm{~Hz})$, and beta-2 $(16-30 \mathrm{~Hz})$ based on our previous studies $[26,32]$. $T$ represents the time interval of interest and a window from 0 to $1000 \mathrm{~ms}$ was selected to reflect the movement stage. $N$ is the number of time-frequency bins in a selected two-dimension rectangular matrix. Following previous literature [22, 24, 32], we extracted averaged ERD powers at two electrodes C3 and C4 to represent the left and right sensorimotor activation, respectively. In accordance with previous studies, an asymmetric index (i.e., no hemispheric effect) was calculated with the following formula and 
used in further statistical analyses [26]. A more positive value indicates more activation of the right sensorimotor area.

Asymmetric index $=($ C3 ERD power $)-($ C4 ERD power $)$

\section{Statistical Analysis}

A statistical analysis was performed using SPSS version 23.0. GraphPad Prism version 7 and custom Matlab scripts were used for the figure visualization. Analysis of variance (ANOVA) was performed separately for each frequency band and the asymmetric index was used as the dependent variable. The level of significance was $p<0.05$. Violation of sphericity was corrected by Green-Geisser. Potential between-group difference of the dependence variable at baseline was tested by independent $t$ tests. Twoway repeated measures analysis of variance (ANOVA) with time (pre vs. post) as a within-subject factor and group (Group 1 vs. Group 2) as a between-subject factor was used to analyze the movement-related ERD. Three-way repeated measures ANOVA with time (baseline vs. post-stimulation) and condition (mirror view vs. direct view) as within-subject factors and group (Group 1 vs. Group 2) as a between-subject factor was used to analyze the MVF-induced ERD. In case of any significant effect found, paired t tests were used for the post hoc comparisons. If any of the dependent variable showed significant betweengroup difference at baseline, analysis of covariance (ANCOVA) with the baseline value as the covariance would be used instead. Missing data were imputed using a last observation carried forward (LOCF) method; that is, if a subject dropped out, the missing value was replaced by the last assessment results.

\section{Results}

Among the twenty included participants, one participant (age $=25$ years, male) in Group 2 dropped out after the first session because he was afraid of the potential risks caused by TBS, although he did not report any side effects after his first session. A demonstration of movement-related ERD and MVFinduced ERD at baseline $(n=20)$ was depicted in Fig. 2.

\section{Movement-related Erd}

The results of an ANOVA examining movement-related ERD are shown in Table 1 and the descriptive data are graphically depicted in Figure 3A. No baseline between-group difference was found in any of the frequency band (all $p>0.05$ ). There were significant time effects noted in both the high $\mathrm{mu}\left(\mathrm{F}_{(1,18)}=\right.$ $\left.6.54, p=0.020, \eta^{2}=0.266\right)$ and low beta bands $\left(F_{(1,18)}=6.00, p=0.025, \eta^{2}=0.249\right)$. A significant interaction effect was noted in the high mu band $\left(F_{(1,18)}=4.47, p=0.049, \eta^{2}=0.199\right)$ and the mean changes ( \pm standard deviation) of asymmetric index in the high mu band were $0.65 \pm 0.47$ in Group 1 and $0.06 \pm 0.75$ in Group 2, indicating that a more obvious shift in sensorimotor high mu ERD toward the right hemisphere was noted in participants who received priming iTBS, compared with those who received 
iTBS alone. Figure 4 shows the topographical distributions of movement-related high mu ERD at baseline and post-stimulation. No other significant effects were found in the two-way ANOVA.

\section{Mvf-induced Erd}

The results of the ANOVA examining MVF-induced ERD are shown in Table 2 and the descriptive data are graphically depicted in Figure 3B. No baseline between-group difference was found in any of the frequency band (all $p>0.05)$. Significant time effects were observed in both the high $\mathrm{mu}\left(\mathrm{F}_{(1,18)}=4.65, p\right.$ $\left.=0.045, \eta^{2}=0.205\right)$ and low beta $\left(F_{(1,18)}=6.10, p=0.024, \eta^{2}=0.253\right)$ bands, and significant condition effects were observed in the low $\mathrm{mu}\left(\mathrm{F}_{(1,18)}=20.84, p<0.001, \eta^{2}=0.537\right)$, high $\mathrm{mu}\left(\mathrm{F}_{(1,18)}=16.12, p=\right.$ $\left.0.001, \eta^{2}=0.472\right)$, and low beta bands $\left(F_{(1,18)}=11.72, p=0.003, \eta^{2}=0.394\right)$. No other significant effects were found in the three-way ANOVA. Within-condition comparisons tested by paired t-tests showed that a dominant right-lateralized sensorimotor ERD was found in the low mu (baseline: $\mathrm{t}=2.78, p=0.012$; poststimulation: $\mathrm{t}=2.47, p<0.001$ ), high $\mathrm{mu}$ (baseline: $\mathrm{t}=2.47, p=0.023$; post-stimulation: $\mathrm{t}=4.35, p<$ 0.001 ), and low beta bands (baseline: $\mathrm{t}=2.31, p=0.032$; post-stimulation: $\mathrm{t}=3.40, p=0.003$ ) under the MVF condition, in contrast to the DVF condition. Significant differences across time were only found in the high $\mathrm{mu}(\mathrm{t}=2.35, p=0.030)$ and low beta bands $(\mathrm{t}=2.79, p=0.012)$ under the MVF condition, but not in other frequency bands under the DVF condition.

\section{Discussion}

The present study aims to elucidate the modulatory effect of priming iTBS on sensorimotor oscillation during voluntary movement and movement observation, in contrast to non-priming iTBS on healthy adults. Our study found that: 1) both stimulation protocols increased movement-related ERD in high mu and low beta bands, with a superior effect in regard to enhancing movement-related high mu ERD in participants who received priming iTBS; and 2) both protocols were equivalent in enhancing MVF-induced ERD in the high mu and low beta bands. Our study indicates that priming iTBS with cTBS seems to yield a similar facilitatory effect on the sensorimotor activation induced by movement and MVF-based observation, as non-priming iTBS.

\section{Movement-related ERD}

Voluntary hand movements could attenuate the activities of mu and beta rhythms, as reported by several human EEG experiments carried out by Pfurtscheller and his colleagues [35, 38, 39]. The power suppression of mu and beta bands over the central electrodes induced by voluntary movement has been confirmed to be correlated with the activation of the sensorimotor area [16]. iTBS is a facilitator of cortical excitability [1]. A priming session of cTBS has been shown to intensify the facilitatory effect of subsequent iTBS on the motor cortex, as suggested by an increased MEP amplitude $[8,11]$ and a reduction of $\mathrm{SICI}[11]$, in contrast to iTBS without priming. In the present study, we found that both 
priming iTBS and non-priming iTBS enhanced movement-related ERD only in high mu and low beta, but not in low mu and high beta bands. Sensorimotor mu ERD has been found to be correlated with both MEP and SICI [40-42]; however, the functional dissociation between low mu and high mu rhythms has been reported by a previous study [43]. The authors found that high mu ERD, but not low mu ERD, had a clear response to movement execution. Indeed, some early studies have demonstrated that movement-related high mu ERD was found to be topographically restricted to the sensorimotor area, while low mu ERD was relatively topographically widespread [38]. Movement-related high mu ERD is likely to be a more sensitive marker of the activation of sensorimotor activation caused by voluntary movement than low mu ERD [16, 43]. This could explain why we could only observe the facilitatory effect of two motor cortex stimulations on high mu but not low mu ERD. Sensorimotor beta ERD during movement is also thought to be correlated with voluntary movement and motor control. In the present study, low beta ERD was increased by both stimulation protocols, while high beta ERD remained stable at pre- and post-stimulation. The impairment of beta oscillation during movement has been found in previous studies on healthy older adults [44] and patients with motor impairments due to a stroke [45]. These studies analyzed beta rhythms from 15 to $30 \mathrm{~Hz}$, thus neglecting the low beta band. Our findings showed that high beta ERD was stable during movement in healthy adults with intact motor functions, after repetitive excitatory motor cortex stimulation, while low beta ERD varied along with the stimulation. Low beta ERD is more likely to be correlated with low-level motor control related to corticomotor excitability, while high beta ERD may be correlated with high-order motor functions, such as cognitive-motor control [46]. In future, the effects of TBS on high beta oscillation and its relationship with the level of motor deficits and the ability of an individual to relearn motor skills warrant investigation in older populations and with patients with neurological conditions, such as stroke.

In this study, priming iTBS seems to be superior to iTBS in enhancing movement related ERD in the high mu band. Although the effect was only modest, we found that a priming session of cTBS did not abolish the excitatory effect of the subsequent iTBS session; it may even potentially boost the effects, found in high mu ERD. These findings support the potential role of metaplasticity in modulating the cortical response to excitatory motor cortex stimulation [6].

\section{MVF-induced ERD}

Previous experiments have found that MVF induced a shift in ERD toward the sensorimotor area ipsilateral to the moving hand, compared with DVF, in healthy adults [22, 24, 32]. In the present study, the effects of MVF were found in low mu, high mu, and low beta bands, which is in line with previous investigations conducted among healthy adults [22, 24, 32]. Moreover, we found that both protocols enhanced the MVF-induced ERD in the high mu and low beta bands, indicating that both TBS protocols delivered to the motor cortex could make the brain more receptive to MVF, which provide neurophysiological evidence to explain the behavioral benefits from excitatory motor cortex stimulation on the observation-based motor learning $[32,47,48]$. However, we did not find any significant difference between the two TBS protocols. MVF-induced ERD is thought to be a summation of the activation of the 
sensorimotor cortex, presumed MNS, and other neural networks related to attention and cognitive control $[25,49]$. The magnitude of MVF-induced ERD may also be influence with the level of perception of embodiment during the observation, thus resulting in greater variability in response [50]. The small between-group differences in the motor area may not be clearly reflected on the MVF-induced ERD. The potentiate effect of priming protocol on MVF-induced oscillation may become observable when it is in combination of observation-based behavioral training (e.g., mirror training) [32]. Moreover, for populations with reduced responses to MVF and a limited ability to relearn motor skills via observation - patients who have suffered a stroke, for example [51] - the alteration of this marker also needs to be examined and correlated with the potential functional recovery caused by priming iTBS.

\section{Limitations}

Our experiment has some limitations. First, the sample size of this study was small and so the study is likely to be underpowered. However, as an exploratory study, there is no similar existing study from which to calculate an appropriate sample size. We followed previous ERD research and simply used an empirically estimated sample size of 10 cases in each group [52]. Second, we did not include behavioral outcomes for evaluation in this study. According to a previous study conducted with healthy adults, the neurophysiological effects of iTBS are less likely to be generalized into real behavioral changes in participants with intact motor functions [32]. Therefore, it would be more meaningful to explore the behavioral outcomes altered by different stimulation protocols in participants with motor deficits - for example, patients with stroke. Thirdly, only two groups (priming iTBS vs. non-priming iTBS) were employed in the present study, since our focus was to find potential differential effects of these two groups. Without a no iTBS control, we cannot rule out the significant time effects might be attributed to spontaneous fluctuations in ERD across different sessions, although the test-retest reliability of sensorimotor ERD has been proven in a previous experiment [53]. Lastly, we investigated different frequency bands separately, since the previous literature has suggested that functional differences exist between them [43]. Thus, we allowed multiple testing on each frequency band separately, without applying a Bonferroni method for a more stringent $p$ value. Together with our previous experiment [32] and other studies $[16,22,43]$, further investigations among healthy adults might focus on high mu and low beta ERD.

\section{Conclusions}

Both priming iTBS and standard iTBS delivered to motor cortex increases in relation to movement-related sensorimotor activation in the hemisphere contralateral to the moving hand and MVF-induced sensorimotor activation in the hemisphere ipsilateral to the moving hand. The latter seems to be only superior in inducing a shift of movement-related sensorimotor activation towards the hemisphere contralateral to the moving hand, as suggested by the increase in high mu ERD.

\section{Equipment:}


1. a. SymAmps2 amplifier and Curry 7, Compumedics Neuroscan, Charlotte, NC, USA

2. b. MagPro X100 and MagOption rTMS stimulator with Coil C-B60 Butterfly, Standard, MagVenture, Denmark

3. c. Localite TMS Navigator, Localite, Germany

\section{Abbreviations}

iTBS

Intermittent Theta Burst Stimulation

cTBS

Continuous Theta Burst Stimulation

EEG

Electroencephalography

MVF

Mirror Visual Feedback

ERD

Event-related Desynchronization

rTMS

Repetitive Transcranial Magnetic Stimulation

MEP

Motor Evoked Potential

LTP

Long-term Potentiation

LTD

Long-term Depression

$\mathrm{SICl}$

Short-interval Intracortical Inhibition

MNS

Mirror Neuron System

RMT

Resting Motor Threshold

AMT

Active Motor Threshold

DVF

Direct Visual Feedback

ERSP

Event-related Spectral Perturbation

ANOVA

Analysis of Variance

ANCOVA 
Analysis of Covariance

LOCF

Last Observation Carried Forward

\section{Declarations}

\section{Ethics approval and consent to participate}

This study was approved by the Human Subjects Ethics Committee, The Hong Kong Polytechnic University (Ref. number: HSEARS20190326003). All participants voluntarily consented to participate in this study and their written informed consent was obtained before participation commenced. The study was performed in accordance with the Declaration of Helsinki.

\section{Consent to publish}

$\mathrm{JZ}$ and KF have approved the submission of the final version of the manuscript. This manuscript did not include any details, images, or videos relating to an individual person.

\section{Availability of data and materials}

The datasets analyzed during the current study are available from the corresponding author on reasonable request.

\section{Competing interests}

None

\section{Funding}

None

\section{Authors' Contributions}

$\mathrm{JZ}$ and KF were involved in the cconceptualisation and design of the study. JZ conducted the experiment and KF supervised the progress. JZ wrote up the first draft of the study. KF reviewed and edited the manuscript. JZ and KF approved the submission of the manuscript.

\section{Acknowledgements}

We thank the University Research Facility in Behavioral and Systems Neuroscience (UBSN), The Hong Kong Polytechnic University, for facility supports. Part of the material in the manuscript was presented 
orally at the International Symposium on Translational Research in Brain Stimulation on Nov 6-10, 2019, Shanghai, China.

\section{References}

1. Huang YZ, Rothwell JC. The effect of short-duration bursts of high-frequency, low-intensity transcranial magnetic stimulation on the human motor cortex. Clin Neurophysiol. 2004;115(5):106975.

2. Huang YZ, Edwards MJ, Rounis E, Bhatia KP, Rothwell JC. Theta burst stimulation of the human motor cortex. Neuron. 2005;45(2):201-6.

3. Karabanov A, Ziemann U, Hamada M, George MS, Quartarone A, Classen J, et al. Consensus Paper: Probing Homeostatic Plasticity of Human Cortex With Non-invasive Transcranial Brain Stimulation. Brain Stimul. 2015;8(5):993-1006.

4. Mc Calley D, Lench D, Doolittle J, Hamilton S, DeVries W, Hanlon C. Effect of Theta-Burst Stimulation Dose on Motor Cortex Excitability: a parametric evaluation of $600,1200,1800$ pulses per session. Brain Stimulation: Basic, Translational, and Clinical Research in Neuromodulation 2019, 12(2):528.

5. Schilberg L, Schuhmann T, Sack AT. Interindividual Variability and Intraindividual Reliability of Intermittent Theta Burst Stimulation-induced Neuroplasticity Mechanisms in the Healthy Brain. J Cogn Neurosci. 2017;29(6):1022-32.

6. Muller-Dahlhaus F, Ziemann U. Metaplasticity in human cortex. Neuroscientist. 2015;21(2):185-202.

7. Hassanzahraee M, Zoghi M, Jaberzadeh S. How different priming stimulations affect the corticospinal excitability induced by noninvasive brain stimulation techniques: a systematic review and meta-analysis. Rev Neurosci 2018.

8. Opie GM, Vosnakis E, Ridding MC, Ziemann U, Semmler JG. Priming theta burst stimulation enhances motor cortex plasticity in young but not old adults. Brain Stimul. 2017;10(2):298-304.

9. Mastroeni C, Bergmann TO, Rizzo V, Ritter C, Klein C, Pohlmann I, et al. Brain-derived neurotrophic factor-a major player in stimulation-induced homeostatic metaplasticity of human motor cortex? PLoS One. 2013;8(2):e57957.

10. Cassidy JM, Gillick BT, Carey JR. Priming the brain to capitalize on metaplasticity in stroke rehabilitation. Phys Ther. 2014;94(1):139-50.

11. Murakami T, Muller-Dahlhaus F, Lu MK, Ziemann U. Homeostatic metaplasticity of corticospinal excitatory and intracortical inhibitory neural circuits in human motor cortex. J Physiol. 2012;590(22):5765-81.

12. Dionísio A, Gouveia R, Duarte IC, Castelhano J, Duecker F, Castelo-Branco M. Continuous theta burst stimulation increases contralateral mu and beta rhythms with arm elevation: implications for neurorehabilitation. J Neural Transm 2019.

13. Tremblay S, Rogasch NC, Premoli I, Blumberger DM, Casarotto S, Chen R, et al. Clinical utility and prospective of TMS-EEG. Clin Neurophysiol. 2019;130(5):802-44. 
14. Cohen MX. Where Does EEGC, From and What Does It Mean? Trends Neurosci 2017, 40(4):208 - 18.

15. Neuper C, Wörtz M, Pfurtscheller G. ERD/ERS patterns reflecting sensorimotor activation and deactivation. In: Event-Related Dynamics of Brain Oscillations. edn.; 2006: 211-22.

16. Pfurtscheller G, Lopes da Silva FH. Event-related EEG/MEG synchronization and desynchronization: basic principles. Clin Neurophysiol. 1999;110(11):1842-57.

17. Frenkel-Toledo S, Bentin S, Perry A, Liebermann DG, Soroker N. Mirror-neuron system recruitment by action observation: effects of focal brain damage on mu suppression. Neuroimage. 2014;87:127-37.

18. Muthukumaraswamy SD, Johnson BW, McNair NA. Mu rhythm modulation during observation of an object-directed grasp. Brain Res Cogn Brain Res. 2004;19(2):195-201.

19. Rizzolatti G, Fadiga L, Gallese V, Fogassi L. Premotor cortex and the recognition of motor actions. Brain Res Cogn Brain Res. 1996;3(2):131-41.

20. Caspers S, Zilles K, Laird AR, Eickhoff SB. ALE meta-analysis of action observation and imitation in the human brain. Neuroimage. 2010;50(3):1148-67.

21. Pineda JA. Sensorimotor cortex as a critical component of an 'extended' mirror neuron system: Does it solve the development, correspondence, and control problems in mirroring? Behav Brain Funct. 2008;4:47.

22. Bartur G, Pratt H, Dickstein R, Frenkel-Toledo S, Geva A, Soroker N. Electrophysiological manifestations of mirror visual feedback during manual movement. Brain Res. 2015;1606:113-24.

23. Rossiter HE, Borrelli MR, Borchert RJ, Bradbury D, Ward NS. Cortical mechanisms of mirror therapy after stroke. Neurorehabil Neural Repair. 2015;29(5):444-52.

24. Lee HM, Li PC, Fan SC. Delayed mirror visual feedback presented using a novel mirror therapy system enhances cortical activation in healthy adults. J Neuroeng Rehabil. 2015;12:56.

25. Zhang JJQ, Fong KNK, Welage N, Liu KPY. The Activation of the Mirror Neuron System during Action Observation and Action Execution with Mirror Visual Feedback in Stroke: A Systematic Review. Neural Plast 2018, 2018:2321045.

26. Fong KN, Ting KH, Chan CC, Li LS. Mirror therapy with bilateral arm training for hemiplegic upper extremity motor functions in patients with chronic stroke. Hong Kong Med J. 2019;25(Suppl 3(1):304.

27. Oldfield RC. The assessment and analysis of handedness: the Edinburgh inventory. Neuropsychologia. 1971;9(1):97-113.

28. Rossi S, Hallett M, Rossini PM, Pascual-Leone A. Screening questionnaire before TMS: an update. Clin Neurophysiol. 2011;122(8):1686.

29. Perellón-Alfonso R, Kralik M, Pileckyte I, Princic M, Bon J, Matzhold C, et al. Similar effect of intermittent theta burst and sham stimulation on corticospinal excitability: A 5-day repeated sessions study. Eur J Neurosci. 2018;48(4):1990-2000.

30. Läppchen CH, Ringer T, Blessin J, Schulz K, Seidel G, Lange R, et al. Daily iTBS worsens hand motor training-a combined TMS, fMRI and mirror training study. Neuroimage. 2015;107:257-65. 
31. Hamzei F, Läppchen CH, Glauche V, Mader I, Rijntjes M, Weiller C. Functional plasticity induced by mirror training: the mirror as the element connecting both hands to one hemisphere. Neurorehabil Neural Repair. 2012;26(5):484-96.

32. Zhang JJ, Fong KNK. Enhancing mirror visual feedback with intermittent theta burst stimulation in healthy adults. Restor Neurol Neurosci 2019.

33. Goldsworthy MR, Pitcher JB, Ridding MC. The application of spaced theta burst protocols induces long-lasting neuroplastic changes in the human motor cortex. Eur J Neurosci. 2012;35(1):125-34.

34. Zhou Q, Tao HW, Poo MM. Reversal and stabilization of synaptic modifications in a developing visual system. Science. 2003;300(5627):1953-7.

35. Pfurtscheller G, Neuper C. Event-related synchronization of mu rhythm in the EEG over the cortical hand area in man. Neurosci Lett. 1994;174(1):93-6.

36. Delorme A, Makeig S. EEGLAB: an open source toolbox for analysis of single-trial EEG dynamics including independent component analysis. J Neurosci Methods. 2004;134(1):9-21.

37. Makeig S. Auditory event-related dynamics of the EEG spectrum and effects of exposure to tones. Electroencephalogr Clin Neurophysiol. 1993;86(4):283-93.

38. Pfurtscheller G, Neuper C, Krausz G. Functional dissociation of lower and upper frequency mu rhythms in relation to voluntary limb movement. Clin Neurophysiol. 2000;111(10):1873-9.

39. Stancak A Jr, Pfurtscheller G. Desynchronization and recovery of beta rhythms during brisk and slow self-paced finger movements in man. Neurosci Lett. 1995;196(1-2):21-4.

40. Takemi M, Masakado Y, Liu M, Ushiba J. Sensorimotor event-related desynchronization represents the excitability of human spinal motoneurons. Neuroscience. 2015;297:58-67.

41. Takemi M, Masakado Y, Liu M, Ushiba J. Event-related desynchronization reflects downregulation of intracortical inhibition in human primary motor cortex. J Neurophysiol. 2013;110(5):1158-66.

42. Thies M, Zrenner C, Ziemann U, Bergmann TO. Sensorimotor mu-alpha power is positively related to corticospinal excitability. Brain Stimul. 2018;11(5):1119-22.

43. Frenkel-Toledo S, Bentin S, Perry A, Liebermann DG, Soroker N. Dynamics of the EEG power in the frequency and spatial domains during observation and execution of manual movements. Brain Res. 2013;1509:43-57.

44. Schmiedt-Fehr C, Mathes B, Kedilaya S, Krauss J, Basar-Eroglu C. Aging differentially affects alpha and beta sensorimotor rhythms in a go/nogo task. Clin Neurophysiol. 2016;127(10):3234-42.

45. Rossiter HE, Boudrias MH, Ward NS. Do movement-related beta oscillations change after stroke? J Neurophysiol. 2014;112(9):2053-8.

46. Adam R, Isabella S, Chan JL. Insight into motor control and motor impairment from stroke and beta oscillations. J Neurophysiol. 2015;114(6):3033-5.

47. von Rein E, Hoff M, Kaminski E, Sehm B, Steele CJ, Villringer A, et al. Improving motor performance without training: the effect of combining mirror visual feedback with transcranial direct current stimulation. J Neurophysiol. 2015;113(7):2383-9. 
48. Hoff M, Kaminski E, Rjosk V, Sehm B, Steele CJ, Villringer A, et al. Augmenting mirror visual feedbackinduced performance improvements in older adults. Eur J Neurosci. 2015;41(11):1475-83.

49. Deconinck FJ, Smorenburg AR, Benham A, Ledebt A, Feltham MG, Savelsbergh GJ. Reflections on mirror therapy: A systematic review of the effect of mirror visual feedback on the brain. Neurorehabil Neural Repair. 2015;29(4):349-61.

50. Alimardani M, Nishio S, Ishiguro H. The Importance of Visual Feedback Design in BCls; from Embodiment to Motor Imagery Learning. PLoS One. 2016;11(9):e0161945.

51. Bartur G, Pratt H, Frenkel-Toledo S, Soroker N. Neurophysiological effects of mirror visual feedback in stroke patients with unilateral hemispheric damage. Brain Res. 2018;1700:170-80.

52. Hasegawa K, Kasuga S, Takasaki K, Mizuno K, Liu M, Ushiba J. Ipsilateral EEG mu rhythm reflects the excitability of uncrossed pathways projecting to shoulder muscles. J Neuroeng Rehabil. 2017;14(1):85.

53. Espenhahn S, de Berker AO, van Wijk BCM, Rossiter HE, Ward NS. Movement-related beta oscillations show high intra-individual reliability. Neuroimage. 2017;147:175-85.

\section{Tables}

Table 1. Difference in movement-related event-related desynchronization between groups at baseline and post-stimulation

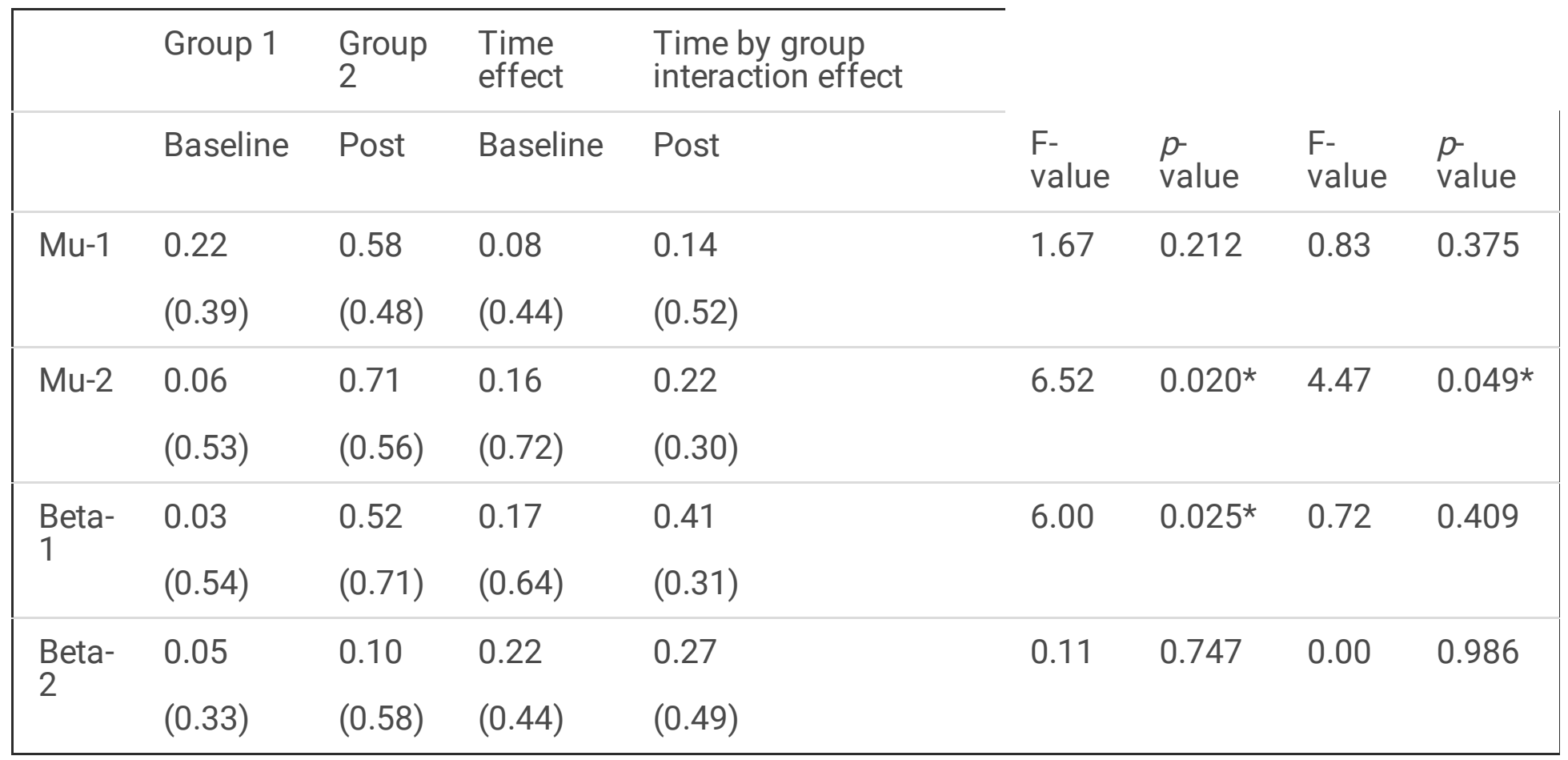

Data are represented as mean (SD); *represents $p<0.05$.

Table 2. Difference in mirror visual feedback-induced event-related desynchronization between groups at baseline and post-stimulation 


\begin{tabular}{|c|c|c|c|c|c|c|}
\hline & & Group 1 & Group 2 & Results 9 & & \\
\hline & & Baseline & Post & Baseline & Post & \\
\hline \multirow[t]{8}{*}{$\mathrm{Mu}-1$} & $M V F$ & 0.31 & 0.44 & 0.10 & 0.56 & (a) $\mathrm{F}=0.99, p=0.332$ \\
\hline & & $(0.35)$ & $(0.75)$ & $(0.90)$ & $(0.84)$ & (b) $\mathrm{F}=1.80, p=0.196$ \\
\hline & & & & & & (c) $\mathrm{F}=20.84, p<0.001^{*}$ \\
\hline & & & & & & (d) $\mathrm{F}=5.34 p=0.474$ \\
\hline & & & & & & (e) $\mathrm{F}=2.14, p=0.160$ \\
\hline & & & & & & (f) $\mathrm{F}=0.03, p=0.863$ \\
\hline & $D V F$ & -0.05 & -0.28 & -0.50 & -0.32 & \\
\hline & & $(0.36)$ & $(0.79)$ & $(0.86)$ & $(0.53)$ & \\
\hline \multirow[t]{8}{*}{ Mu-2 } & $M V F$ & 0.12 & 0.71 & 0.14 & 0.67 & (a) $\mathrm{F}=4.65, p=0.045^{\star}$ \\
\hline & & $(0.51)$ & $(0.92)$ & $(1.28)$ & $(1.13)$ & (b) $\mathrm{F}=0.17, p=0.898$ \\
\hline & & & & & & (c) $\mathrm{F}=16.12, p=0.001^{*}$ \\
\hline & & & & & & (d) $\mathrm{F}=0.64, p=0.434$ \\
\hline & & & & & & (e) $\mathrm{F}=2.74, p=0.115$ \\
\hline & & & & & & (f) $\mathrm{F}=0.16, p=0.692$ \\
\hline & $D V F$ & -0.24 & -0.19 & -0.64 & -0.44 & \\
\hline & & $(0.46)$ & $(0.76)$ & $(0.89)$ & $(0.68)$ & \\
\hline \multirow[t]{8}{*}{ Beta-1 } & $M V F$ & -0.11 & 0.53 & 0.03 & 0.45 & (a) $\mathrm{F}=6.10, p=0.024^{*}$ \\
\hline & & $(0.51)$ & $(0.64)$ & $(0.84)$ & $(0.96)$ & (b) $\mathrm{F}=0.60, p=0.450$ \\
\hline & & & & & & (c) $\mathrm{F}=11.72, p=0.003^{*}$ \\
\hline & & & & & & (d) $\mathrm{F}=0.25, p=0.626$ \\
\hline & & & & & & (e) $\mathrm{F}=3.63, p=0.073$ \\
\hline & & & & & & (f) $\mathrm{F}=0.00, p=0.996$ \\
\hline & $D V F$ & -0.36 & -0.10 & -0.37 & -0.32 & \\
\hline & & $(0.43)$ & $(0.67)$ & $(0.74)$ & $(0.70)$ & \\
\hline \multirow[t]{4}{*}{ Beta-2 } & $M V F$ & -0.11 & 0.10 & 0.16 & 0.08 & (a) $\mathrm{F}=2.55, p=0.626$ \\
\hline & & $(0.30)$ & $(0.25)$ & $(0.28)$ & $(0.37)$ & (b) $\mathrm{F}=0.96, p=0.340$ \\
\hline & & & & & & (c) $\mathrm{F}=3.28, p=0.087$ \\
\hline & & & & & & (d) $\mathrm{F}=0.78, p=0.639$ \\
\hline
\end{tabular}




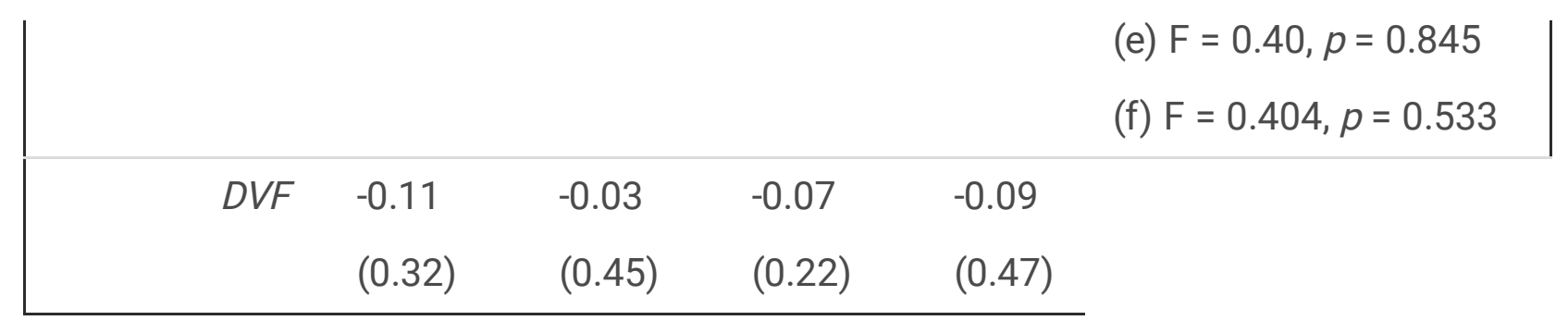

१ Repeated measures ANOVA; data are represented as mean (SD); *represents $p<0.05$

(a) Time effect; (b) Time by group interaction effect; (c) Condition effect; (d) Condition by group interaction effect; (e) Time by condition interaction effect; ( $f$ ) Time by condition by group interaction effect

\section{Figures}

(A) Left-index finger tapping

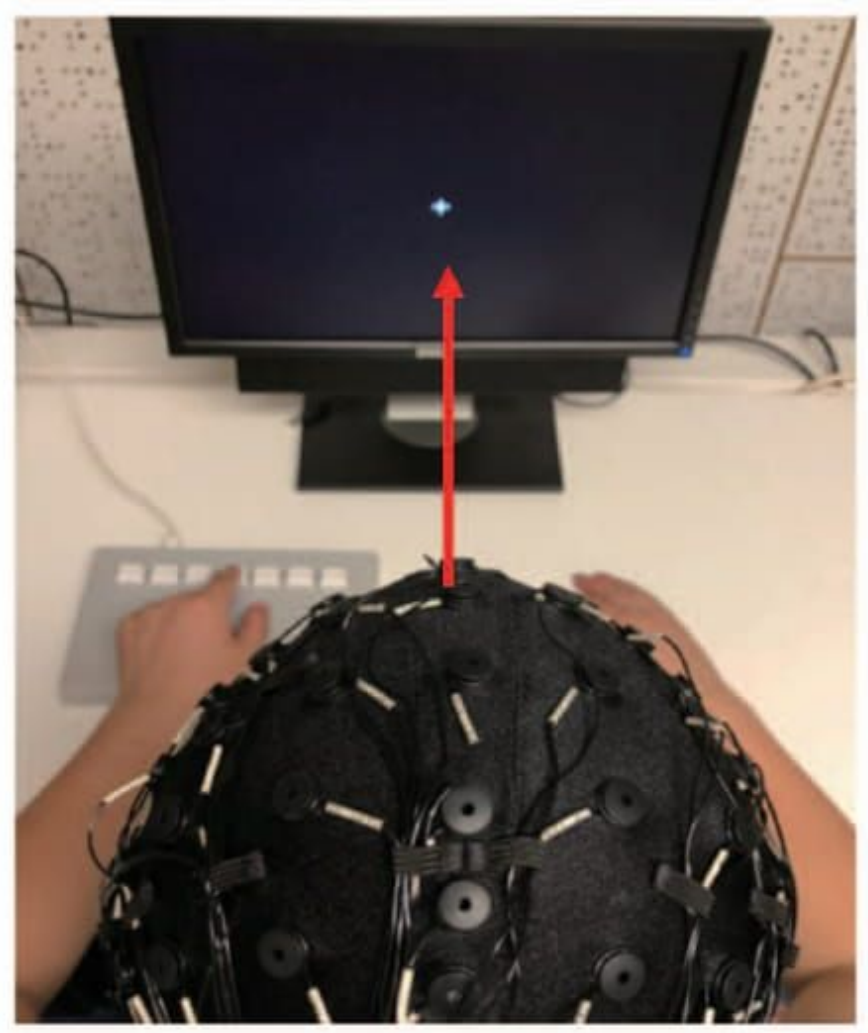

(B) Right-index finger tapping with MVF

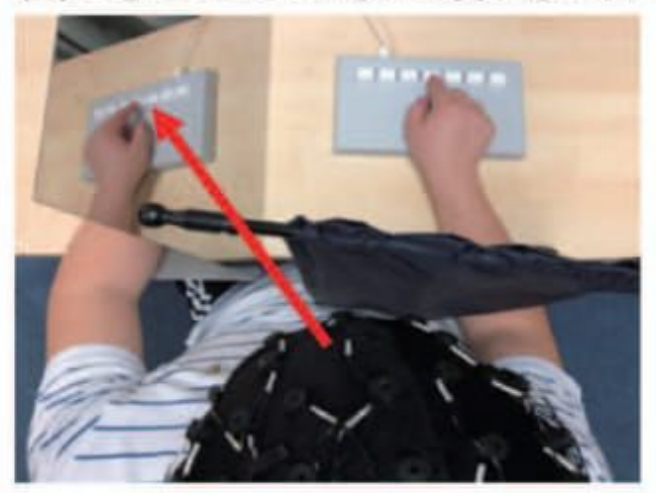

(C) Right-index finger tapping with MVF

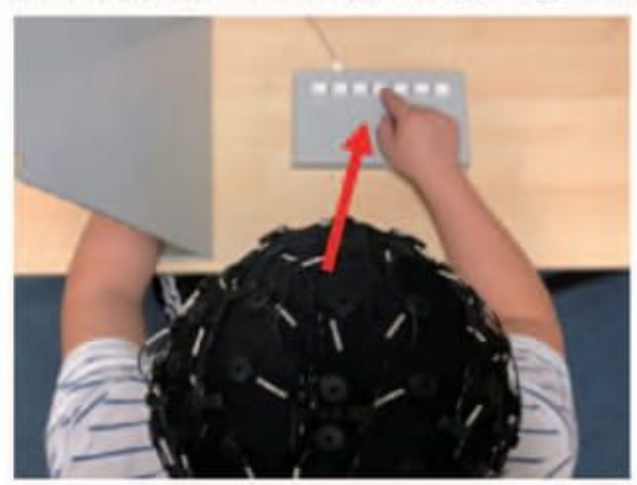

\section{Figure 1}

Setup of the EEG experiment. (A) Participants performed left-index finger tapping in response to auditory cues. Participants were instructed to focus on a fixation cross. (B) Participants performed right-index finger tapping in response to auditory cues. Participants were instructed to observe the mirror visual feedback of their moving index finger. A black curtain was used to block the direct view of their moving hand (C) Participants performed right index tapping in response to auditory cues. Participants were 
instructed to observe their moving index finger. The red arrows represent the visual direction during the movement.

Left-index finger tapping
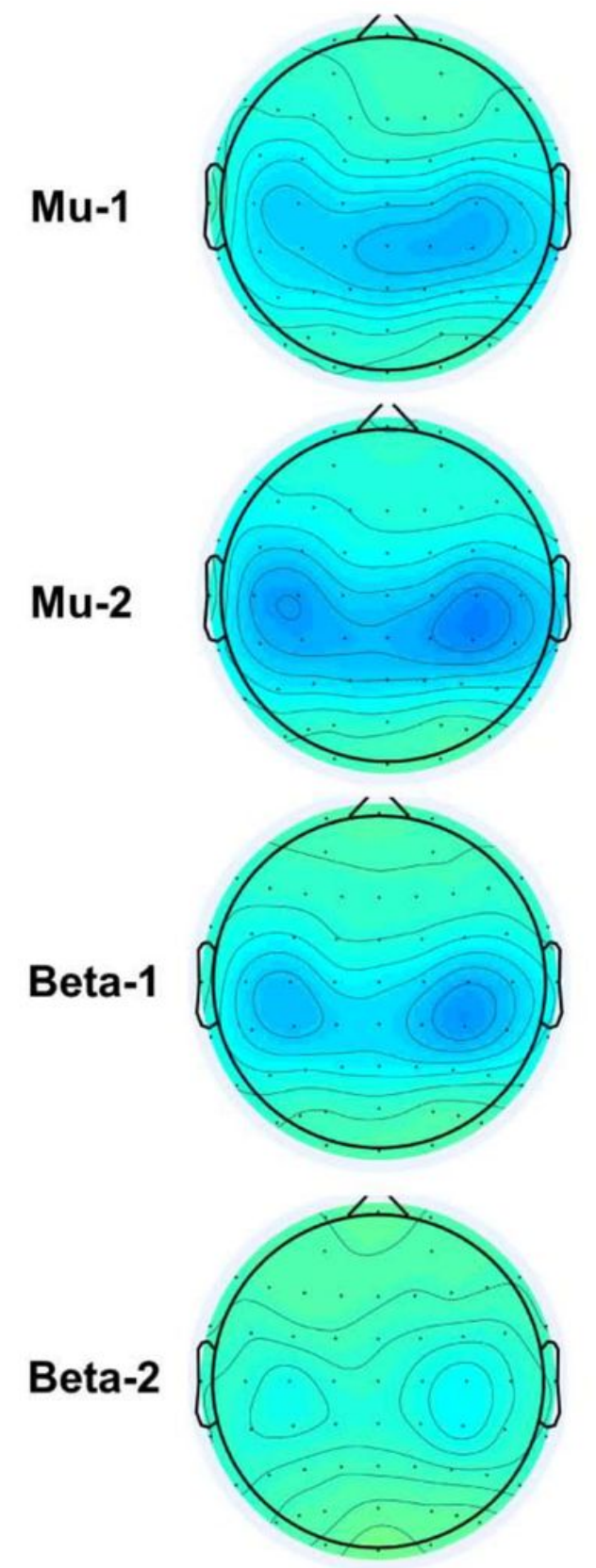

Right-index finger tapping with MVF finger tapping with DVF
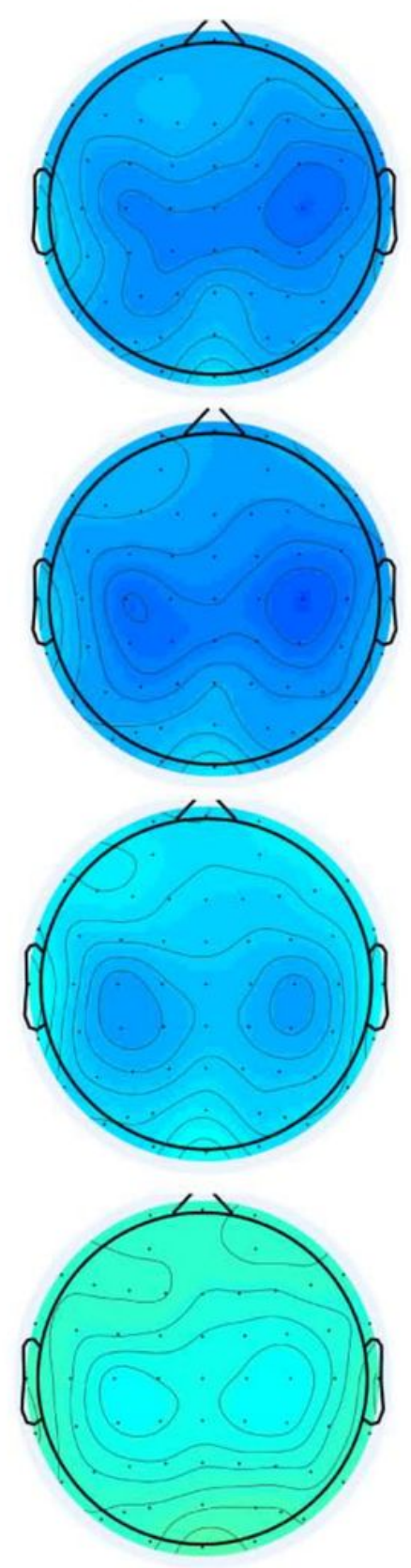

\section{Right-index}
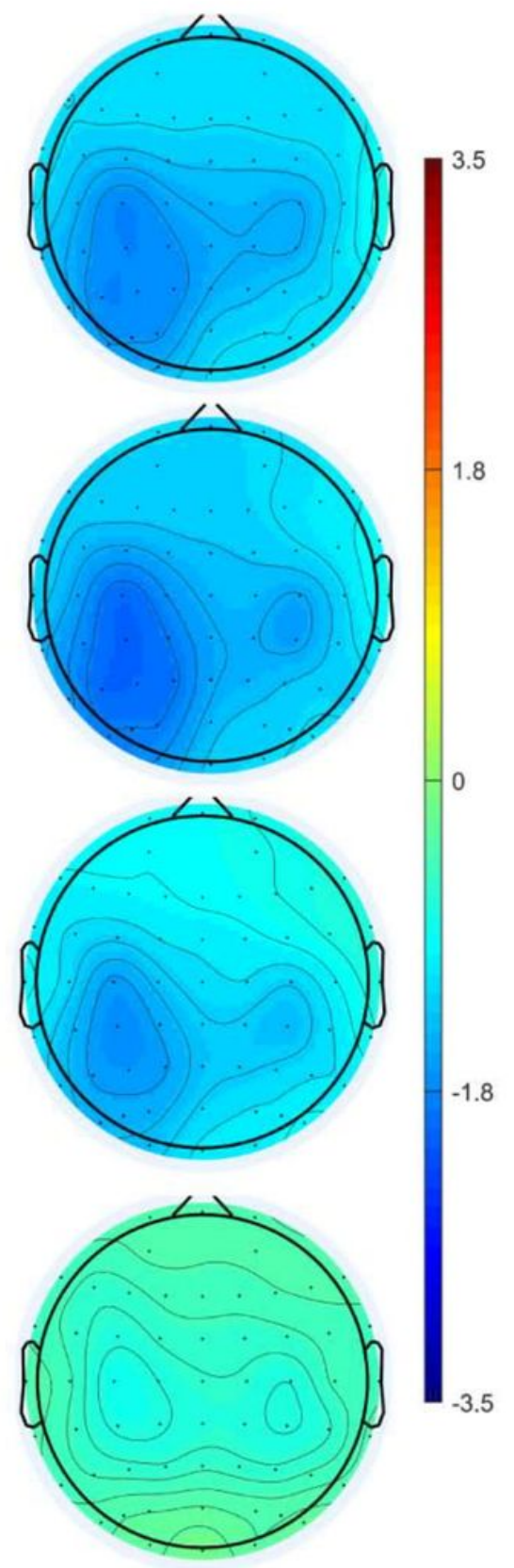

Figure 2

Demonstration of sensorimotor ERD at baseline $(n=20)$ 

(A)
Movement-related ERD $\quad *$ Group 1

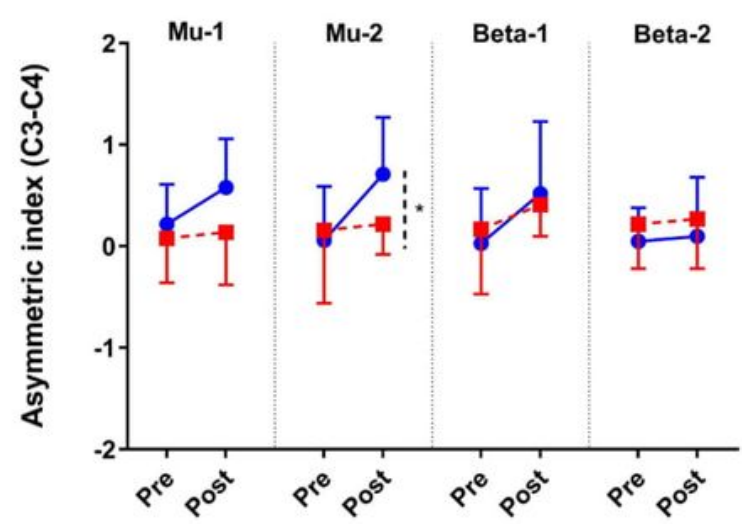

(B) MVF-induced ERD $:$ Group 1
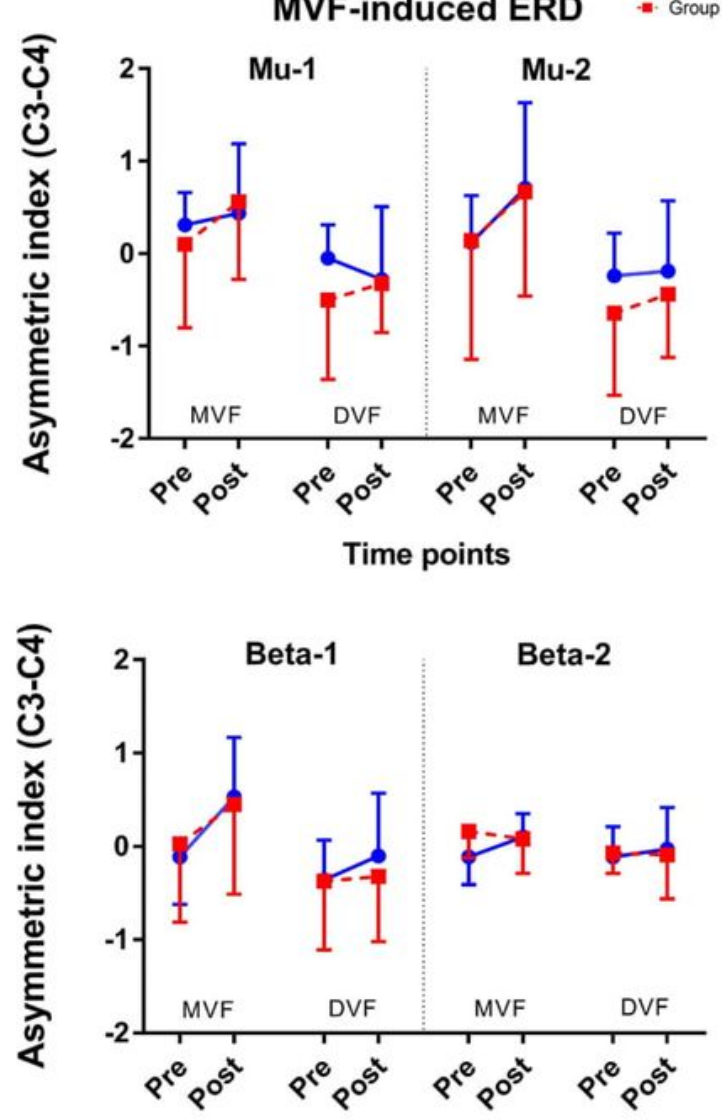

Time points

\section{Figure 3}

Change of asymmetric index. (A) Movement-related ERD and (B) MVF-induced ERD * represents $p$ for the interaction effect $<0.05$ 

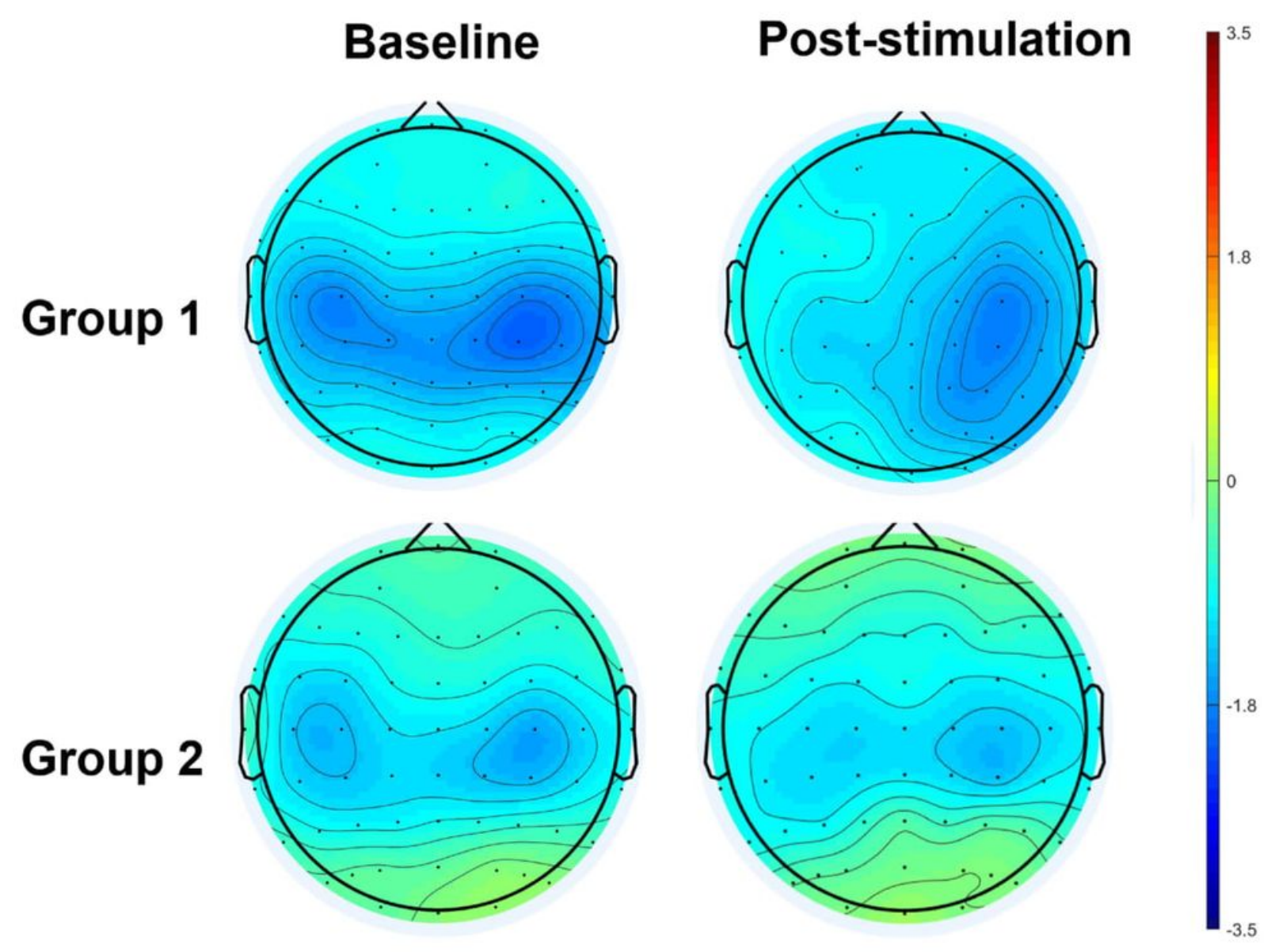

Figure 4

Topographical distribution of movement-related high mu ERD at baseline and post-stimulation. A significant interaction effect favoring Group 1 was observed, in contrast to Group 2. 